\title{
LIMITED APPROXIMATION OF NUMERICAL RANGE OF NORMAL MATRIX
}

\author{
MARIA ADAM AND JOHN MAROULAS
}

Abstract. Let $A$ be an $n \times n$ normal matrix, whose numerical range $N R[A]$ is a $k$-polygon. If a unit vector $v \in W \subseteq \mathbb{C}^{n}$, with $\operatorname{dim} W=k$ and the point $v^{*} A v \in \operatorname{Int} N R[A]$, then $N R[A]$ is circumscribed to $N R\left[P^{*} A P\right]$, where $P$ is an $n \times(k-1)$ isometry of $\{\operatorname{span}\{v\}\}_{W}^{\perp} \rightarrow \mathbb{C}^{n}$, [1]. In this paper, we investigate an internal approximation of $N R[A]$ by an increasing sequence of $N R\left[C_{s}\right]$ of compressed matrices $C_{s}=R_{s}^{*} A R_{s}$, with $R_{s}^{*} R_{s}=I_{k+s-1}, \quad s=1,2, \ldots, n-k$ and additionally $N R[A]$ is expressed as limit of numerical ranges of $k$-compressions of $A$.

Mathematics subject classification (2000): 15A18, 15A60, 47A20.

Keywords and phrases: Compression, eigenvalue, numerical range.

\section{REFERENCES}

[1] M. Adam and J. Maroulas, On compressions of normal matrices, Linear Algebra Appl., 341 (2002), 403-418.

[2] M. ADAM AND P. PSARRAKOS, On a compression of normal matrix polynomials, Linear and Multilinear Algebra, 52, 3-4 (2004), 251-263.

[3] H.-L. GAU AND P.Y. WU, Numerical range of a normal compression, Linear and Multilinear Algebra, 52, 3-4 (2004), 195-201.

[4] H.-L. GAU AND P.Y. Wu, Numerical range of a normal compression II, Linear Algebra Appl., 390 (2004), 121-136.

[5] R.A. Horn And C.R. Johnson, Topics in Matrix Analysis, Cambridge University Press, Cambridge, 1991. 